\title{
Radiocarbon
}

\author{
1972
}

\section{ATOMIC ENERGY RESEARCH INSTITUTE OF KOREA RADIOCARBON MEASUREMENTS II}

\author{
KYUNG RIN YANG \\ Radiocarbon Dating Laboratory, \\ Atomic Energy Research Institute, Seoul, Korea
}

$\mathrm{C}^{14}$ measurements of archaeologic and geochemical samples using sample synthesized benzene in a Beckman LS-100 liquid scintillation spectrometer are reported. Rather high background counting rates of ca. 8 to $9 \mathrm{cpm}$ and counting efficiency of ca. 45 to $48 \%$ for 4 cc counting vial of potassium free glass are observed. The counting vial (total volume of $10 \mathrm{cc}$ ) is supported by an aluminum bar for geometry control. Background and standard counting rates measured during the past 17 months are listed in Tables 1 and 2, respectively. Slight seasonal variations were observed.

Carbon in the sample was precipitated as calcium carbonate and then converted to carbon dioxide-lithium carbide-acetylene-benzene (Tamers, 1965; Noakes, Kim, and Akers, 1967). Organic carbon sample was pretreated first with hydrochloric acid and then with sodium hydroxide before combustion, the resulting carbon dioxide was converted to calcium carbonate. All reactions were done with a vacuum chemical train at -25 to -28 in. $\mathrm{Hg}$ pressure consisting of an organic carbon combustion system, an acid digestion vessel, a gas purification column, 3 tanks of 3 L. gas storage, 3 gas transfer traps for liquid nitrogen or dry ice, a stainless steel reaction chamber for carbide formation and acetylene generation, and a catalyst column. Vacuum of the chemical train and gas pressure generated in the chemical train were checked by 5 vacuum pressure gauges. Lithium metal in shot form was used to convert carbon dioxide to carbide and vanadium alumina catalyst was used for trimerization of acetylene. Vanadium alumina catalyst was activated at $300^{\circ} \mathrm{C}$ for ca. 4 hours before being in contact with acetylene. Over-all chemical recovery of carbon in the sample was ca. $80 \%$. Measurements were duplicated for archaeologic samples when enough sample was supplied. Minimum synthesized benzene from samples up to 20,000 years old is 1 cc for accurate measurement. When enough sample was supplied, $3 \mathrm{cc}$ of synthesized benzene and 1 cc of benzene phosphor cocktail were taken for the activity measurement. The resulting counting solution contains $0.3 \%$ PPO and $0.02 \%$ POPOP.

Age calculations are based on a $\mathrm{C}^{14}$ half-life of 5568 years and $95 \%$ of the activity of the NBS oxalic acid standard. Errors quoted refer only to the standard deviation calculated from statistical analysis of sample 
and background counting rates. Data listed here are not corrected for isotopic fractionation.

\section{ACKNOWLEDGMENTS}

The author expresses his gratitude to Stephen M. Kim, P. K. Sohn, and B. S. Han for their generous technical help. Thanks are extended to C. K. Pak, H. S. Chang, and K. B. Choi for preparing and measuring samples. The laboratory is financed by the A. E. R. I., Korea.

\section{SAMPLE DESCRIPTIONS}

\section{ARCHAEOLOGIC SAMPLES}

\section{AERIK-8. Sokchang-ni, Loc. 1, YM-4}

\section{$20,830 \pm 1880$}

\section{8,880 B.c.}

Charcoal and ashes from hearth of upper Palaeolithic habitation floor of stratified Sokchang-ni, Loc. 1. (AERIK-5: R., 1970, v. 12, p. 351) at Kum R. terrace, Changki-myon, Kongju-kun, Chungchongnam-do, Korea $\left(36^{\circ} 21^{\prime} \mathrm{N}\right.$ Lat, $127^{\circ} 10^{\prime} \mathrm{E}$ Long). Coll. 1970 and subm. by P. K. Sohn, Yonsei Univ. Mus., Seoul, Korea. Comment (P.K.S.): consistent and seems accurate.

\section{Shido series}

A shell mound and cairn at Shido-ri, Pukdo-myon, Buchonkun, Kyunggi-do, Korea (37 $32^{\prime} \mathrm{N}$ Lat, $126^{\circ} 26^{\prime} \mathrm{E}$ Long) contained many

Table 1

Counter* Backgrounds for $\mathrm{C}^{14 * *}$

\begin{tabular}{lrcc}
\hline Date & C.P.M. & Eff. $(\%) \dagger$ & D.P.M. \\
\hline Feb., 1970 & $8.34 \pm 0.04$ & 45.78 & $18.22 \pm 0.09$ \\
Mar. & $8.54 \pm 0.06$ & 45.45 & $18.79 \pm 0.13$ \\
Apr. & $8.46 \pm 0.07$ & 46.15 & $18.33 \pm 0.15$ \\
May & $9.01 \pm 0.07$ & 46.40 & $19.42 \pm 0.15$ \\
June & $9.26 \pm 0.08$ & 47.12 & $19.65 \pm 0.17$ \\
July & $10.44 \pm 0.09$ & 47.72 & $21.88 \pm 0.19$ \\
Aug. & $9.49 \pm 0.08$ & 47.64 & $19.92 \pm 0.17$ \\
Sept. & $8.87 \pm 0.07$ & 47.88 & $18.53 \pm 0.15$ \\
Oct. & $9.07 \pm 0.09$ & 47.29 & $19.18 \pm 0.19$ \\
Nov. & $9.09 \pm 0.08$ & 44.96 & $20.22 \pm 0.18$ \\
Dec. & $8.78 \pm 0.08$ & 47.64 & $18.43 \pm 0.17$ \\
Jan., 1971 & $8.88 \pm 0.07$ & 47.21 & $18.81 \pm 0.15$ \\
Feb. & $8.73 \pm 0.10$ & 48.13 & $18.14 \pm 0.21$ \\
Mar. & $8.59 \pm 0.09$ & 47.30 & $18.16 \pm 0.19$ \\
Apr. & $8.22 \pm 0.09$ & 47.38 & $17.35 \pm 0.19$ \\
May & $8.41 \pm 0.09$ & 47.17 & $17.83 \pm 0.19$ \\
June & $8.59 \pm 0.08$ & 47.64 & $18.03 \pm 0.17$ \\
\hline
\end{tabular}

* Liquid scintillation counting system, Beckman LS-100.

* 4 cc spectrograde benzene contains $0.3 \%$ PPO and $0.02 \%$ POPOP.

$\dagger$ Obtained from One-to-One Quench Correction Curve. 


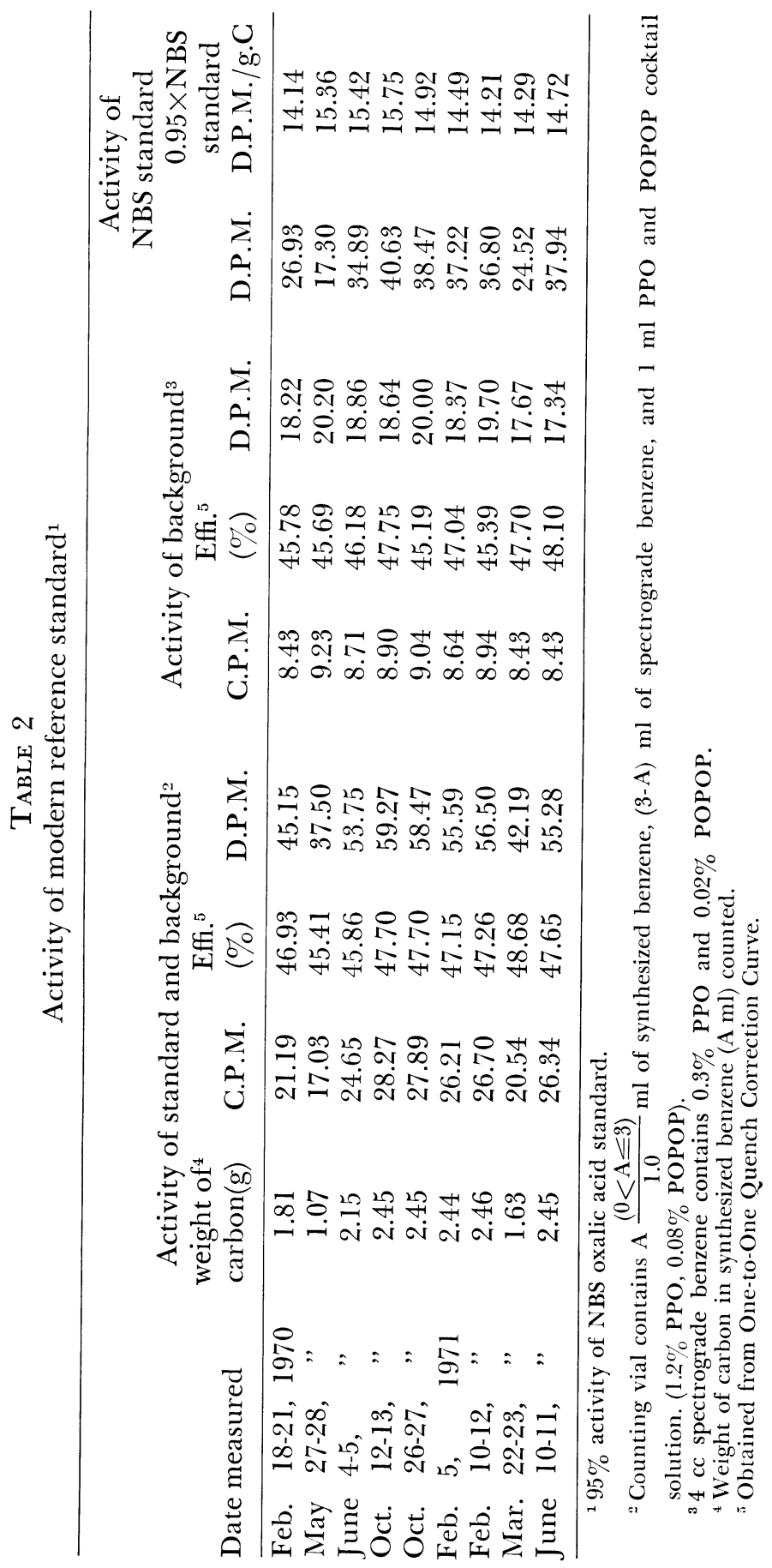


pieces of comb-pattern, plain-coarse, and Kimhae pottery from Neolithic to Early Iron cultures. Shido is an island ca. $4 \mathrm{~km}^{2}$ near W coast of central Korea. Coll. 1970 and subm. by B. S. Han, Natl. Mus. of Korea.

AERIK-9. Shido, Loc. I, No. 1

$1980 \pm 60$

AERIK-9. Shido, Loc. I,

Charcoal from mingled shell crust layer of shell mound, ca. $30 \mathrm{~cm}$ below surface with Kimhae pottery.

AERIK-10. Shido, Loc. I, No. 2

$2470 \pm 60$

Charcoal from mingled shell crust layer of shell mound, ca. $60 \mathrm{~cm}$ below surface with plain-coarse pottery.

AERIK-11. Shido, Loc. I, No. 3

$3040 \pm 60$

Charcoal from black soil layer under mingled shell crust layer of shell mound, ca. $100 \mathrm{~cm}$ below surface with comb-pattern pottery.

AERIK-12. Shido, Loc. II, No. 1

$2870 \pm 60$

Charcoal from stone layer of cairn with comb-pattern pottery.

920 B.C.

\section{$3100 \pm 60$}

AERIK-13. Shido, Loc. II, No. 2

1150 B.c.

Charcoal from base of cairn with comb-pattern pottery.

AERIK-14. Shido, Loc. II, No. 3

$3040 \pm 60$

Charcoal from base of cairn with comb-pattern pottery.

1090 B.C.

AERIK-15. Kosong site

$1730 \pm 70$

Charcoal from black humus below shell crust layer of shell mound at Dongwai-dong, Kosong-up, Kyungsangnam-do, Korea $\left(34^{\circ} 58^{\prime} \mathrm{N}\right.$ Lat, $128^{\circ} 20^{\prime}$ E Long). Assoc. with Kimhae pottery, animal bone implements, and earthen ware coll. 1969 and subm. by B. S. Han.

AERIK-16. Songwon-ni, YM-5

$2880 \pm 120$

930 B.c.

Charcoal from Songwon-ni on Kum R. bank, Changki-myon, Kongjukun, Chungchongnam-do, Korea ( $36^{\circ} 27^{\prime} \mathrm{N}$ Lat, $127^{\circ} 15^{\prime}$ E Long). Assoc. with plain-coarse pottery and polished stone artifacts. Coll. 1970 and subm. by P. K. Solnn. Comment (P.K.S.): later date probably due to much natural and human disturbance.

AERIK-17. Songpa-dong, YM-6

$1920 \pm 130$

A.D. 30

Wood from Songpa-dong at S bank of Han R., Songdong-ku, Seoul, Korea ( $37^{\circ} 30^{\prime} \mathrm{N}$ Lat, $127^{\circ} 06^{\prime} \mathrm{E}$ Long). Assoc. with elaborate wooden structure several tens of meters long. Coll. 1970 by Y. S. Kim, Dong-A 
Ilbo, Daily Newspaper Co., Seoul, Korea, and subm. by P. K. Sohn. Comment (P.K.S.): date seems good in view of archaeologic chronology.

\section{AERIK-18. Sokchang-ni, Loc. 1, YM-7}

$2990 \pm 130$

Charcoal and ashes from humus layer of Loc. 1, Sokchang-ni, (AERIK-5, -8: R., 1970, v. 12, p. 351; this list). Sample from a concentrated hearth-like spot, previously heavily disturbed by cultivation assoc. with undisturbed chipped stone implements. Coll. 1971 and subm. by P. K. Sohn. Comment (P.K.S.): date too young; contamination seems apparent.

\section{Guri Cave series}

Samples from Guri Cave, Quezon, Palawan, Philippines $\left(9^{\circ} 16^{\prime} \mathrm{N}\right.$ Lat, $117^{\circ} 58^{\prime}$ E Long) were dated. Coll. 1970 by R. B. Fox, Natl. Mus. of the Philippines and subm. by P. K. Sohn. Comment (R.B.F.): agrees with archaeologic data.

Charcoal from depth ca. $212 \mathrm{~cm}$ at Sq. 103-B; assoc. with shells and flake assemblage found in front of cave, probably Level $\mathrm{B}$.

AERIK-20. Guri Cave, Catalog No. 62-p-2235

$$
\begin{array}{ll} 
& \mathbf{8 1 3 0} \pm \mathbf{1 8 0} \\
\text { Outer fraction } & \mathbf{6 1 8 0} \text { B.c. } \\
& \mathbf{7 8 9 0} \pm \mathbf{9 0} \\
\text { Inner fraction } & \mathbf{5 9 4 0} \text { B.C. }
\end{array}
$$

Shell from Epi-Paloeolithic site, assoc. with Paloeolithic tool tradition persisting into post-Pleistocene period. Sample from Level B, depth ca. 124 to $137 \mathrm{~cm}$ below datum point. Comment: figures suggest no isotopic replacement.

\section{AERIK-21. Songpa-dong}

$1440 \pm 70$

Wood from ca. $5 \mathrm{~m}$ below surface at Han R., Songpa-dong (AERIK17, above), part of roof construction materials. Coll. 1971 and subm. by B. S. Han.

\section{Tongsam-dong series}

Large shell mound from sea shore, Tongsam-dong, Yongdo-ku, Pusan, Korea $\left(35^{\circ} 04^{\prime} \mathrm{N}\right.$ Lat, $129^{\circ} 05^{\prime} \mathrm{E}$ Long) consists of 4 layers involving 3 periods of Neolithic culture (J. Arch. Soc. Korea, 1969, v. 2, p. 3-4) Lowest, Layer 4, contained yunkimun pottery, Layer 3, comb-pattern pottery, and Layer 2, plain-coarse pottery. Excavations made 1969-1971 by Natl. Mus. of Korea. Samples coll. 1971 from Layers 2 and 3 assoc. with pottery, stone artifacts, and animal bone tools. Subm. by B. S. Han. 
AERIK-22. Tongsam-dong, Layer 2

$4170 \pm 100$

Charcoal from Pit HXII, ca. $140 \mathrm{~cm}$ below surface.

2220 B.C.

AERIK-23. Tongsam-dong, Layer 3, No. 1

$4020 \pm 100$

Charcoal from Pit GXII, ca. $140 \mathrm{~cm}$ below surface.

AERIK-24. Tongsam-dong, Layer 3, No. 2 2070 B.C.

Charcoal from Pit HXII, ca. $140 \mathrm{~cm}$ below surface.

$3980 \pm 100$

2030 B.C.

AERIK-25. Tongsam-dong, Layer 3, No. 3

$3930 \pm 100$

1980 B.C.

Charcoal from Pit HXIII, ca. $160 \mathrm{~cm}$ below surface.

AERIK-26. Tongsam-dong, Layer 3, No. 4

$3880 \pm 100$

1930 B.C.

Charcoal from Pit HXIII, ca. $170 \mathrm{~cm}$ below surface.

$4400 \pm 90$

AERIK-27. Tongsam-dong, Layer 3, No. 5

2450 B.C.

Charcoal from Pit HXVII, ca. $150 \mathrm{~cm}$ below surface.

\section{Chunsong series}

Charcoal from dwelling site in Naepyung-ri, Puksan-myon, Chunsong-kun, Kangwon-do, Korea (37 $56^{\prime} \mathrm{N}$ Lat, $127^{\circ} 54^{\prime}$ E Long). Coll. 1971 and subm. by B. S. Han.

AERIK-28. Chunsong, No. 1

$2290 \pm 60$

Charcoal from ca. $40 \mathrm{~cm}$ below surface.

AERIK-29. Chunsong, No. 2

$2930 \pm 60$

Charcoal from ca. $70 \mathrm{~cm}$ below surface.

980 B.C.

AERIK-30. Chunsong, No. 3

$2590 \pm 60$

Charcoal from ca. $70 \mathrm{~cm}$ below surface.

640 B.c.

\section{GEOCHEMICAL SAMPLES}

\section{Atmospheric carbon dioxide series, Seoul, Korea}

Atmospheric $\mathrm{CO}_{2}$ samples were coll. on the roof of A.E.R.I. main building in NE suburb of Seoul city (37 $38^{\prime} \mathrm{N}$ Lat, $127^{\circ} 06^{\prime} \mathrm{E}$ Long) from Feb. 1970 by exposing 1.5 L. $0.5 \mathrm{~N} \mathrm{NaOH}$ in a $900-\mathrm{cm}^{2}$-tray for ca. 5 days (R., 1970, v. 12, p. 467). Samples were precipitated as $\mathrm{CaCO}_{3}$ and $\mathrm{C}^{14}$ activity was measured in the form of synthesized $\mathrm{C}_{6} \mathrm{H}_{6}$, as for archaeologic samples.

Comment: data show higher concentrations of $\mathrm{C}^{14}$ in the rainy season, June and July (Fairhall and Young, 1968). A clear Suess effect was observed in winter. No $\mathrm{C}^{13}$ corrections were made. 


\begin{tabular}{|c|c|c|}
\hline Sample & Date $(1970)$ & $\delta \mathrm{C}^{14}(\% 0)^{*}$ \\
\hline AERIK-31 & Feb. $\quad 2-$ Feb. 7 & $624 \pm 4$ \\
\hline AERIK-32 & Feb. $23-$ Feb. 28 & $454 \pm$ \\
\hline AERIK-33 & Mar. 10 - Mar. 16 & $652 \pm 5$ \\
\hline AERIK-34 & Mar. 25 - Mar. 30 & $553 \pm$ \\
\hline AERIK-35 & Apr. $11-$ Apr. 16 & $688 \pm$ \\
\hline AERIK-36 & Apr. $25-$ Apr. 30 & $617 \pm$ \\
\hline AERIK-37 & May 11 - May 16 & $582 \pm$ \\
\hline AERIK-38 & May $25-$ May 30 & $660 \pm$ \\
\hline AERIK-39 & June $10-$ June 15 & $723 \pm$ \\
\hline AERIK-40 & June $25-$ June 30 & $709 \pm$ \\
\hline AERIK-41 & July $10-J u l y ~ 15$ & $716 \pm$ \\
\hline AERIK-42 & July 25 - July 30 & $716 \pm 5$ \\
\hline AERIK-43 & Aug. $\quad 8$-Aug. 14 & $631 \pm 6$ \\
\hline AERIK-44 & Aug. $25-$ Aug. 31 & $695 \pm 5$ \\
\hline AERIK-45 & Sept. $10-$ Sept. 16 & $702 \pm 20$ \\
\hline AERIK-46 & Sept. $25-$ Sept. 30 & $652 \pm 5$ \\
\hline AERIK-47 & Oct. $10-$ Oct. 15 & $716 \pm 5$ \\
\hline AERIK-48 & Oct. 25 - Oct. 31 & $681 \pm 7$ \\
\hline AERIK-49 & Nov. $25-$ Nov. 30 & $582 \pm 6$ \\
\hline AERIK-50 & Dec. $10-$ Dec. 15 & $653 \pm 6$ \\
\hline AERIK-51 & Dec. 24 - Dec. 30 & $539 \pm 9$ \\
\hline
\end{tabular}

* Above modern reference.

\section{AERIK-52. Atmospheric CO}

$\mathbf{8 1 6} \pm \mathbf{5} \delta \mathbf{C}^{14}(\%)$

Atmospheric $\mathrm{CO}_{2}$ was coll. at Jeju city, Pukjeju-kun, Jeju-do, Korea $\left(33^{\circ} 30^{\prime} \mathrm{N}\right.$ Lat, $126^{\circ} 31^{\prime} \mathrm{E}$ Long) from Aug. 11-14, 1970 by exposing $\mathrm{NaOH}$ solution.

\section{AERIK-53. Rice}

$809 \pm 6 \delta \mathbf{C}^{14}(\%)$

Rice grown at Yoju-kun, Kyunggi-do, Korea $\left(37^{\circ} 18^{\prime} \mathrm{N}\right.$ Lat, $127^{\circ} 38^{\prime}$ E Long) in 1970. Comment: compared with Seoul series and AERIK-52, above, metropolitan area was contaminated by fossil $\mathrm{CO}_{2}$.

\section{REFERENCES}

Fairhall, A. W. and Young, J. A., 1968, Radiocarbon in the environment: RLO-2091-2, Washington Univ., Seattle, $21 \mathrm{p}$.

Kim, W. Y., 1969, Carbon dates in Korean archaeology: Kogohak (J. Arch. Soc. Korea), v. 2 , p. 1-16.

Noakes, J. E., Kim, S. M., and Akers, L. K., 1967, Recent improvements in benzene chemistry for radiocarbon dating: Geochim. et Cosmochim. Acta, v. 31, p. 1094 1096.

Tamers, M. A., 1965, Routine carbon-14 dating using liquid scintillation techniques: Sixth internatl. conf. radiocarbon and tritium dating Proc., Pullman, Washington, June 7-11, 1965, Conf-650652, p. 53-67.

Vogel, J. C., 1970, Groningen radiocarbon dates 1X: Radiocarbon, v. 12, p. 444-471.

Yang, K. R., 1970, Atomic Energy Research Institute of Korea radiocarbon measurements I: Radiocarbon, v. 12, p. 350-352. 